\title{
Case Studies of Machine Bases as Structure-borne Sound Sources in Buildings
}

\author{
B.M. Gibbs and A.T. Moorhouse \\ Liverpool School of Architecture and Building Engineering, University of Liverpool, Liverpool L69 3BX, UK
}

\begin{abstract}
A review is given of recent approaches to the characterisation of structure-borne sound sources, which can have application to the case of machines in buildings. Two primary issues are identified. The first is that the structural dynamics of the receiving structure must be known for a full description of the transmission process and the second concerns the large amount of data required for characterisation and prediction of emission. In order to reduce the data required and thus simplify the analysis and prediction, it is necessary to consider initially, representative installations in order to establish a hierarchy of contact and component excitations and thereby eliminate the least significant. This can be done in several ways: modelling idealised sources and receivers, using measured or theoretical source and receiver data, prior to assembly, and by in-situ measurement of the installed machine. In this paper, case studies are presented of machine bases rigidly connected to concrete floors or steel plates, where a combination of the above methods is used to identify the important transmission paths and the data required for approximating the total transmission. In addition, it is demonstrated that visual inspection of point force mobilities can establish which of the mechanisms, mass, stiffness or resonance are controlling mobility and thereby lead to simplifications.
\end{abstract}

\footnotetext{
* This paper is a revised version of a paper presented by the first author at the Fifth International Congress on Sound and Vibration, Adelaide, December 1997
}

\section{INTRODUCTION}

The appearance of an international standard for sources of structure-borne sound has highlighted rather than solved the problem of estimating structure-borne emission from and source characterisation of vibrating machines and machine components. ${ }^{1}$ The standard gives procedures for measuring the velocities at machine contact points. Although it is limited to resiliently mounted machinery, the velocities obtained often closely approximate those of the free source i.e. obtained when the source operates under 'normal' conditions but is not attached to supporting and connected structures. These data are a subset of those required but they cannot give a complete source characterisation or sufficient information for structure-borne emission. The additional data required will be obtained through an as yet incomplete set of international standards for the measurement of mechanical mobility. ${ }^{2}$

The term characterisation has been used in three ways: 1) when representing the internal mechanisms that produce the vibrations, 2) as a measure of the energy of the vibrating machine and 3) of the ability of the machine to emit power to connected and supporting structures. An example of the first is the pseudo-force method of Verheij et al. where the internal mechanisms that produce the vibrational field on the machine casing or at the contacts are modelled by a substitute set of external point forces. ${ }^{3,4}$ The approach has the advantage of being applicable to cases where sources are strongly coupled and integrated into larger machine assemblies, and cannot be considered in isolation. An example of the second is the surface equivalent source power of Ohlrich, also applicable to integrated assemblies where the source cannot be treated in isolation. ${ }^{5}$ The surface power is the product of the equivalent forces and the corresponding real part of the point mobilities. This is a descriptor of the power generated in the machine by the internal mechanisms but does not lead to transmission predictions. An example of the third is the terminal source power, of Olhrich et al., which has been used to predict transmission from a main gearbox to a helicopter fuselage. ${ }^{6,7}$

Two primary issues are identified. The first is that the structural dynamics of the receiving structure must be known in some form for a description of the transmission process and the second concerns the large amount of data required for characterisation and prediction of emission.

\section{STRUCTURE-BORNE TRANSMISSION}

The first issue can be introduced by considering a single contact and component of excitation. The structure-borne power $W$, when a source $S$ is connected to a receiver $R$, is given by,

$$
W=\frac{1}{2} \frac{\left|v_{s s}\right|^{2}}{\left|Y_{S}+Y_{R}\right|^{2}} \operatorname{Re}\left[Y_{R}\right]
$$

where $v_{s f}$ is the velocity of the free source, $Y_{S}$ is the mobility of the source and $Y_{R}$ is that of the receiver. Three quantities are required to predict structure-borne power, including receiver mobility, which is seldom available since the details of all installations may not be known. Manufacturers of electric motors, for example, expect their products to be bolted to the internal frame of washing machines, the base plate of fans or form part of circulation pumps, etc. ${ }^{9}$ Therefore, the source must be characterised in terms of source parameters only.

Structure-borne sound sources can be characterised, on a power basis (also see Ohlrich et al. ${ }^{6,7}$ ), using free velocity and 